

XII ${ }^{\text {ìmes }}$ Journées Nationales Génie Côtier - Génie Civil

Cherbourg, 12-14 juin 2012

DOI:10.5150/jngcgc.2012.006-E（C) Editions Paralia CFL

disponible en ligne - http://www.paralia.fr - available online

\title{
Dynamique de la zone de swash : influence de la marée et de la morphologie sur les paramètres du run-up
}

\section{Camille ENJALBERT ${ }^{1}$, Nadia SENECHAL ${ }^{1}$, Karin R. BRYAN ${ }^{2}$, ${\text { Giovanni } \mathrm{COCO}^{3} \text {, Jamie MACMAHAN }}^{4}$, Jenna BROWN ${ }^{4}$}

1. Université Bordeaux 1, UFR Sciences de la Terre et de la Mer, UMR 5805 CNRS EPOC, bat. B18, avenue des facultés, 33705 Talence, France.

c.enjalbert@epoc.u-bordeaux1.fr,n.senechal@epoc.u-bordeaux1.fr

2. Department of Earth and Ocean Science, University of Waikato, Nouvelle Zélande. kbryan@waikato.ac.nz

3. Institute of Environmental Hydraulics, University of Cantabria, Santander, Espagne. giovanni.coco@unican.es

4. Oceanography department, Naval Postgraduate School, Monterey, California, USA. jhmacmah@nps.edu,jenna.a.brown@gmail.com

\section{Résumé :}

L’influence de la pente de plage et de la morphologie sur le run-up sont étudiées, au cours d'un cycle de marée dans des conditions dissipatives, à partir de données vidéo. Le run-up est dominé par la composante infragravitaire qui présente une variation d’un facteur deux au cours du cycle de marée qui ne peut s'expliquer uniquement par une variabilité du forçage au large. Une forte variabilité de la pente de plage est observée et, contrairement aux études précédentes, le run-up n'est pas relié linéairement à $\mathrm{H}_{0}$. La forme du profil de plage ainsi que l'évolution des vagues dans la zone de surf sont significativement corrélées au run-up.

\section{Mots-clés :}

Run-up - Imagerie vidéo - Marée - Paramètres environnementaux - Pente de plage Bande infragravitaire - Conditions dissipatives

\section{Abstract:}

The impact of tide and morphology on run-up parameters in dissipative conditions is assessed, using high-frequency video observations. The infragravity run-up is dominant and shows variations of about $60 \%$ during an entire tidal cycle. This behavior cannot be explained by the evolution of offshore wave conditions. Wave conditions in the surf zone and the beach slope are tidally modulated and significantly correlated to the runup. The role of the shape of the beach profile is also investigated.

Key-words:

Run-up - Video imaging - Dissipative conditions - Infragravity frequency - Beach slope 


\section{Introduction}

Le swash représente la dynamique de la ligne d'eau à l'interface océan-rivage. Le terme run-up désigne les fluctuations de cette ligne d'eau autour d'une valeur moyenne, le setup. Le run-up est le principal processus impliqué dans l'érosion du haut de plage et les problèmes de submersion marine, d'autant plus lors de conditions énergétiques. Sa dynamique varie en fonction de paramètres hydrodynamiques (conditions de vagues) et environnementaux (pente de plage) et est généralement reliée au paramètre d’Irribaren :

$$
\xi_{0}=\frac{\tan \beta}{\sqrt{H_{0} / L_{0}}}
$$

avec $\beta=$ pente de plage, $H_{0}$ et $L_{0}=$ hauteur et longueur d'onde des vagues au large (m), respectivement. Lors de conditions dissipatives, la dynamique du swash est généralement dominée par la composante infragravitaire du run-up $(0.005 \mathrm{~Hz}<f<0.05 \mathrm{~Hz})$ (RUESSINK et al., 1998 ; RUGGIERO et al., 2004) alors que lors de conditions réflectives, la composante incidente $(0.05 \mathrm{~Hz}<f<0.25 \mathrm{~Hz})$ domine (GUZA \& THORNTON, 1982 ; STOCKDON et al., 2006). Des incertitudes demeurent quant à la paramétrisation du run-up en fonction des différents paramètres environnementaux. De récentes études ont notamment montré que la marée et la morphologie ont une influence sur le comportement du run-up (GUEDES et al., 2011). Cependant, l'impact de ces paramètres reste encore peu connu, notamment lors de conditions énergétiques. Nous souhaitons ici étudier la dynamique du run-up lors de conditions énergétiques, en se focalisant sur l’influence de la marée et la morphologie, à partir de données vidéo. Le rôle de facteurs tels la hauteur des vagues dans la zone de surf et la forme du profil de plage est également abordé.

\section{Matériel et méthodes}

\subsection{Site d'étude}

La plage du Truc Vert se situe sur la côte Atlantique, en Gironde. Elle est localisée à environ $10 \mathrm{~km}$ au Nord de l'embouchure du Bassin d'Arcachon et environ $90 \mathrm{~km}$ au Sud de l'estuaire de la Gironde (figure 1) et n'est donc ni influencée par l'hydrodynamique de l'estuaire ni par celle du Bassin d'Arcachon. Elle se situe dans une zone non anthropisée et sa dynamique est donc essentiellement contrôlée par des facteurs naturels. Cette plage sableuse est de type intermédiaire et constitue un système plage-dune complet associé à un double système de barres (CASTELLE et al., 2007). Le régime de marée est de type méso-macrotidal, semi-diurne avec des amplitudes moyennes de 3,7 m en vives-eaux et 1,8 m en mortes-eaux. La plage du Truc Vert est soumise à une dynamique importante avec des hauteurs de vagues annuelles moyennes de 1,4 m et périodes associées de 6,5 s (BUTEL et al., 2002). 


\section{XII ${ }^{\text {èmes }}$ Journées Nationales Génie Côtier - Génie Civil \\ Cherbourg, 12-14 juin 2012}
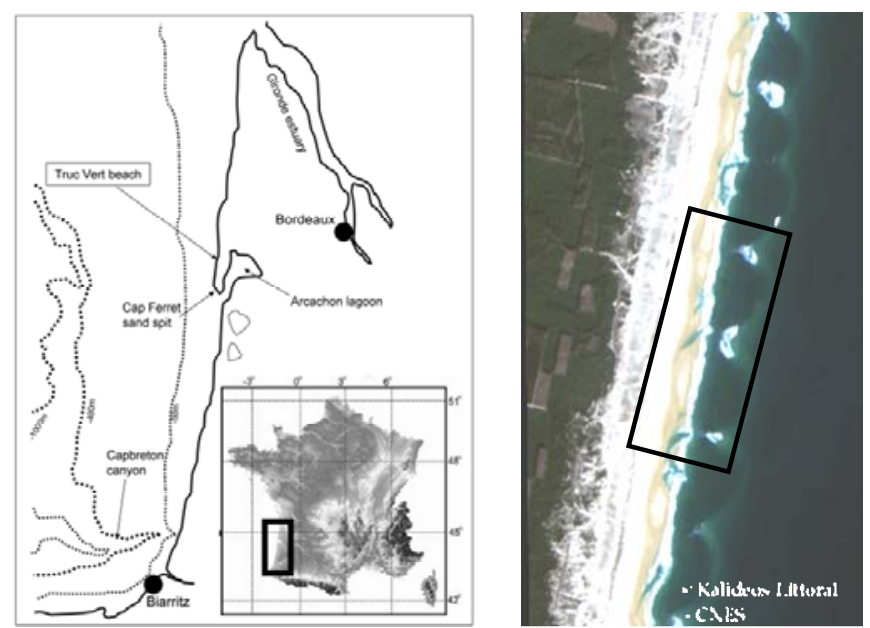

Figure 1. Localisation du site d'étude : plage du Truc Vert, Gironde.

\subsection{Données}

Les données présentées ici ont été acquises dans le cadre de la campagne ECORS -Truc Vert 2008 (SENECHAL et al., 2011a). Le forçage au large a été mesuré par une bouée houle directionnelle située à $20 \mathrm{~m}$ de profondeur en face de la zone d'étude, à environ 1,5 km de la plage (SHOM). Une ligne de capteurs de pression piézorésistifs a été déployée par la Naval Postgraduate School sur la barre interne et a ainsi permis d'évaluer l'évolution des vagues en zone de surf ainsi que d'estimer plus précisément l'altitude du plan d'eau. Des relevés topographiques journaliers ont été réalisés à marée basse à l'aide d'un GPS cinématique dont la résolution est de $10 \mathrm{~cm}$. Durant la campagne, un système de caméras vidéo a été installe en haut de la dune située à l'arrière de la plage a une hauteur par rapport au niveau de marée moyen de $24 \mathrm{~m}$. Il comportait deux caméras haute résolution permettant un enregistrement à haute fréquence (2 Hz) continu durant la journée. Les deux caméras étaient orientées de façon à couvrir une distance dans la direction parallèle à la plage de $800 \mathrm{~m}$ en zone intertidale, et $2 \mathrm{~km}$ en zone subtidale.

A partir des images vidéo, des images timestack ont été générées au droit des capteurs de pression, le long de transects perpendiculaires à la plage (figure 2). La position de la ligne d'eau a été détectée de manière semi-automatique en se basant sur la différence d'intensité des pixels à l'interface entre l'eau et le sable (figure 2). Les valeurs d'élévation du run-up ont été obtenues par redressement géométrique des images vidéo et le signal obtenu a été rectifié à l'aide d'un profil de plage interpolé à partir des données topographiques brutes mesurées. La hauteur du run-up a été estimée par intégration du spectre d'élévation (équation 2) par pas de 15 minutes sur les différentes bandes de fréquence afin de déterminer les composantes incidente $(0.05 \mathrm{~Hz}<f<0.24 \mathrm{~Hz})$ et infragravitaire $(0.004 \mathrm{~Hz}<f<0.05 \mathrm{~Hz})$. La hauteur des vagues en zone de surf a été estimée de manière similaire à partir des données issues des capteurs de pression. 


$$
R=4 * \sqrt{\sum P S D(f) d f}
$$

avec $R=$ =élévation du run-up (m), $P S D=$ spectre de densité d'énergie du run-up. Afin de raccorder les données à la littérature (RUGGIERO et al., 2004; STOCKDON et al., 2006 ; SENECHAL et al., 2011b), la pente de plage a été estimée à partir de la pente linéaire calculée sur une zone définie par \pm 2 fois l'écart type de l'excursion du run-up.
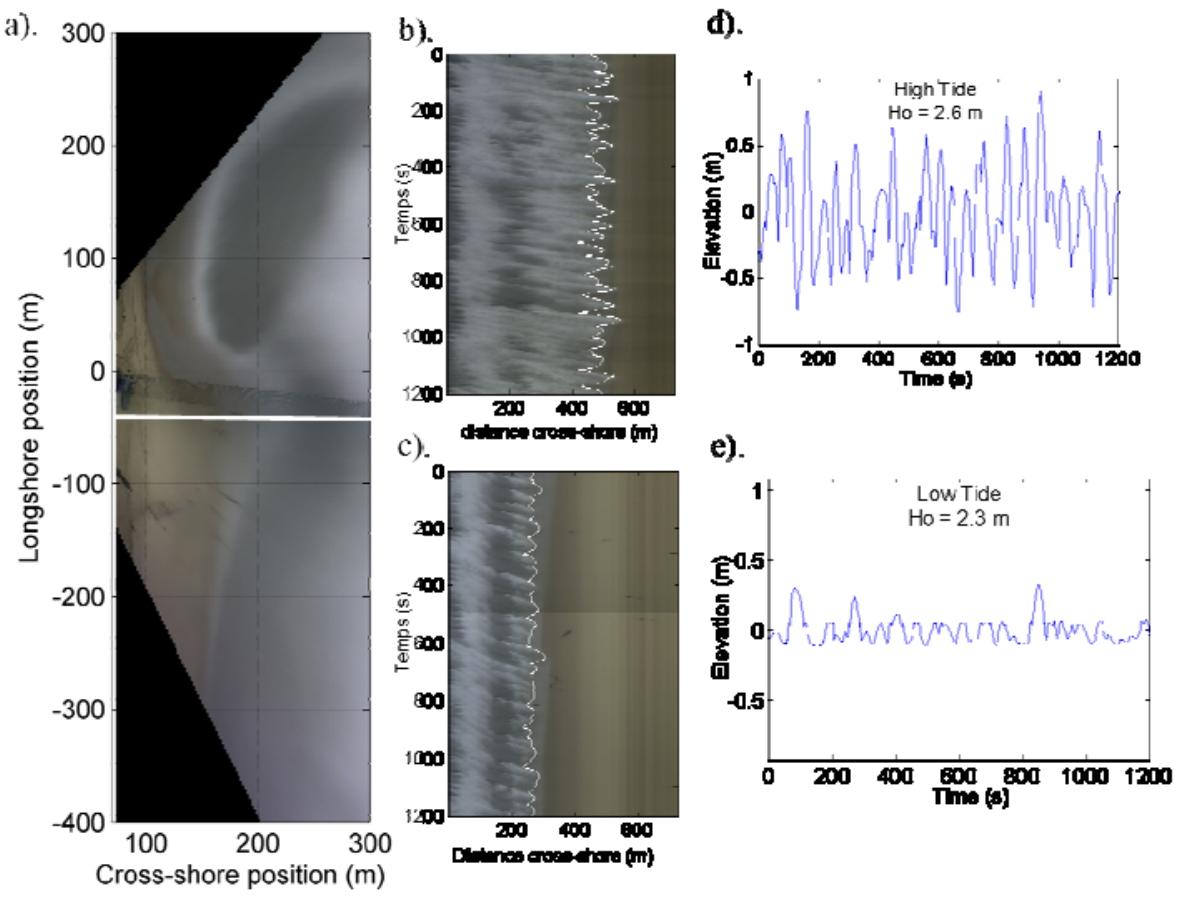

Figure 2. a). Image vidéo moyenne du 27 mars 2008 à marée basse et transect étudié ; images timestack et détection du swash à marée haute (b.) et basse (c.); signaux d'élévation du swash à marée haute (d.) et basse (e.).

Les données présentées ci-après concernent la journée du 27 mars 2008, au cours d’un cycle de marée complet le long d'un transect. La hauteur des vagues au large et la période de pic associée étaient respectivement de $2.4 \mathrm{~m}$ et $10 \mathrm{~s}$ avec un marnage de $2 \mathrm{~m}$.

\section{Résultats}

La figure 3 représente les conditions hydrodynamiques et environnementales ainsi que l'élévation du run-up dans les différentes bandes d'énergie. Une synthèse des conditions hydrodynamiques et environnementales ainsi que les données statistiques d'élévation du run-up sont présentées dans les tableaux 1 et 2 . 

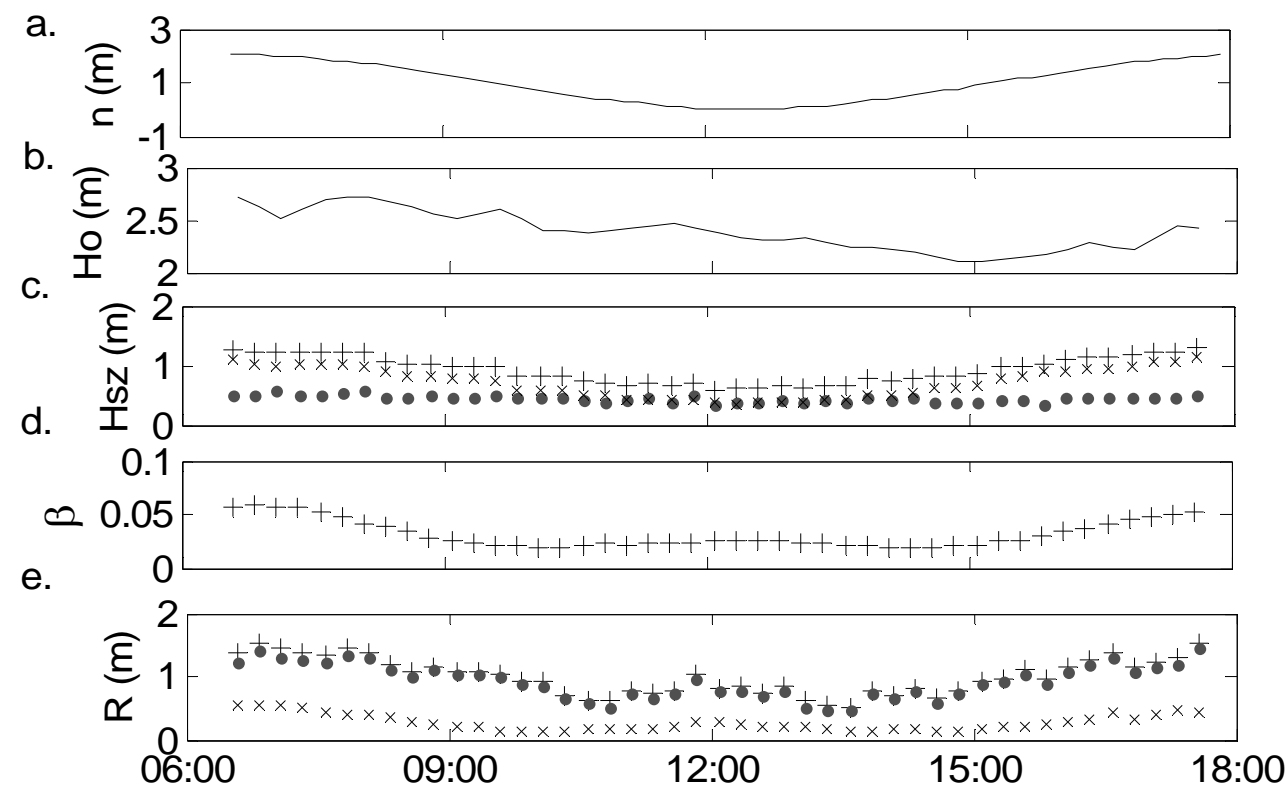

Figure 3. Paramètres hydrodynamiques et environnementaux le 27 mars 2008. a) hauteur d'eau moyenne $(\mathrm{m})$; b) hauteur des vagues au large $(\mathrm{m}) ; c$ ) hauteur des vagues dans la zone de surf $(m)$ selon différentes bandes d'énergie : totale $(+)$, incidente $(0.05-0.25 \mathrm{~Hz})(\times)$ et infragravitaire $(0.005-0.05 \mathrm{~Hz})($.$) ; d) pente de plage ; e)$ Elévation du run-up $(\mathrm{m})$ selon les différentes bandes d'énergie.

Les conditions de vagues au large, énergétiques, sont restées relativement stationnaires au cours de la journée avec une variation maximale de $0.6 \mathrm{~m}$ (soit 22\%) (tableau 1). Le run-up présente une variation beaucoup plus importante, de l'ordre de 60\% (tableau 2) qui touche plus particulièrement la composante infragavitaire qui est majoritairement dominante (tableau 2). Ceci est en accord avec les conditions dissipatives caractérisées par un paramètre d'Iribarren faible $\left(\xi_{\sigma}<0.3\right.$, tableau 1$)$ et les précédentes études (HOLMAN \& SALLENGER, 1985 ; STOCKDON et al., 2006). Dans la littérature, les observations montrent généralement une relation linéaire entre le run-up et le forçage au large (GUZA \& THORNTON, 1982 ; RUESSINK et al., 1998 ; RUGGIERO et al., 2004). Dans le cas présent, la variabilité du run-up ne semble cependant pas pouvoir être imputée uniquement au forçage au large, suggérant l'influence d'autres paramètres. La hauteur des vagues en zone de déferlement ainsi que la pente de plage présentent une modulation tidale importante avec des variations d'un facteur deux au cours du cycle de marée (figure 3, panels c et d respectivement). Au niveau de la barre interne, l'énergie incidente est majoritairement dominante (70\%) et l'énergie infragravitaire reste relativement faible (figure 3, panel c). Ces variations n'expliquent pas la chute observée au niveau de la bande infragavitaire du signal d'élévation du run-up. L'hypothèse d'un contrôle par des paramètres locaux, en particulier la pente de plage est explorée, notamment au travers de la figure 4. 
Tableau 1. Synthèse des conditions hydrodynamiques et environnementales moyennes aux différents stades de marée

\begin{tabular}{lllllll}
\hline Heure & marée $(\mathrm{m})$ & $H_{0}(\mathrm{~m})$ & $T_{p}(\mathrm{~s})$ & $H_{s z}(\mathrm{~m})$ & $\beta$ & $\xi_{0}$ \\
\hline $06 \mathrm{~h} 30$ & 1,89 & 2,52 & 9,96 & 1,20 & 0,05 & 0,36 \\
$12 \mathrm{~h} 30$ & 0,88 & 2,33 & 9,98 & 0,92 & 0,02 & 0,41 \\
18 h 30 & 0,09 & 2,34 & 10,04 & 0,67 & 0,02 & 0,17 \\
\hline
\end{tabular}

Tableau 2. Synthèse des valeurs d'élévation du run-up et part des run-up infragravitaire et incident

\begin{tabular}{llllll}
\hline & $R_{\text {tot }}(m)$ & $R_{\text {ig }}(m)$ & $R_{\text {inc }}(m)$ & $R_{\text {ig }}(\%)$ & $R_{\text {inc }}(\%)$ \\
\hline moyenne & 1,01 & 0,94 & 0,27 & 0,92 & 0,08 \\
écart-type & 0,29 & 0,27 & 0,13 & 0,04 & 0,04 \\
minimum & 0,52 & 0,48 & 0,13 & 0,84 & 0,02 \\
maximum & 1,54 & 1,44 & 0,56 & 0,98 & 0,16 \\
\hline
\end{tabular}

Tel que suggéré dans de précédentes études (RUESSINK et al., 1998 ; RUGGIERO et al., 2004 ; STOCKDON et al., 2006) le run-up présente des comportements distincts en fonction du paramètre d’Irribaren (figure 4). La rupture de pente observée confirme la séparation admise pour la valeur $\xi_{0}=0.3$ et les données sont cohérentes avec les résultats observés dans la littérature. Cependant, il n existe pas d équation commune aux différents auteurs, ce qui confirme l'hypothèse selon laquelle la paramétrisation du runup en fonction du paramètre d'Irribaren serait spécifique à chaque site et aux conditions d'études (RUESSINK et al., 1998 ; SENECHAL et al., 2011b).

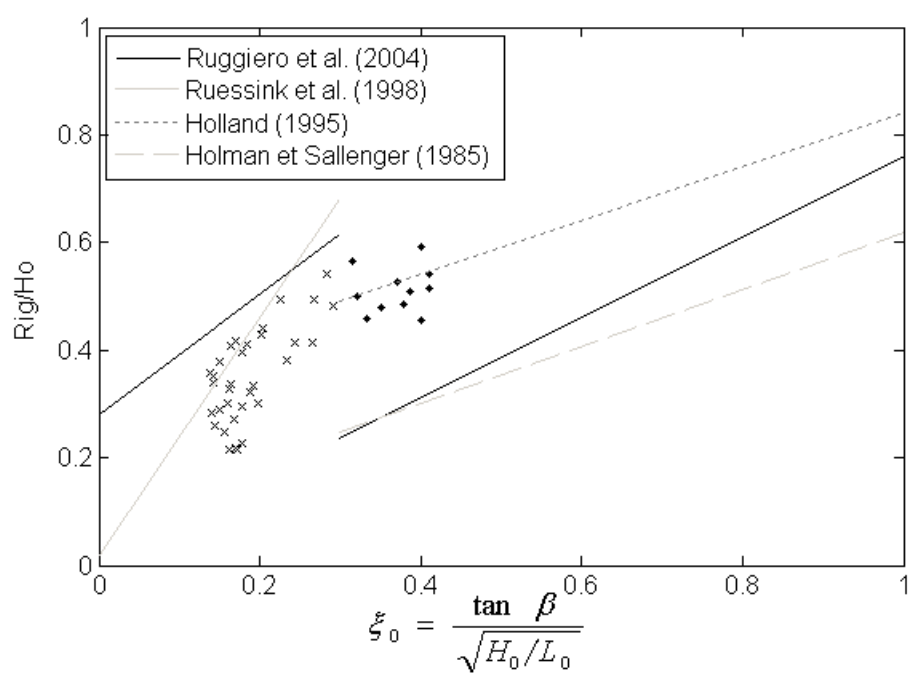

Figure 4. Paramétrisation du run-up infragravitaire normalisé par la hauteur des vagues au large en fonction du paramètre d'Irribaren à marée basse (x) et haute (.). 


\section{XII ${ }^{\text {èmes }}$ Journées Nationales Génie Côtier - Génie Civil \\ Cherbourg, 12-14 juin 2012}

\section{Discussion}

Bien que peu explorée jusqu'alors, la modulation tidale observée dans le comportement du run-up a déjà été reportée dans la littérature (STOCKDON et al., 2006 ; GUEDES et al., 2011). Cependant, il semble que différents paramètres entrent en jeu dans cette variation de comportement (vagues dans la zone de déferlement, pente de plage) dont les influences ne sont pas clairement établies. L’évolution du run-up est contrôlée par le déferlement des vagues dans la zone de surf et la pente de plage qui présente une modulation tidale dont la tendance est similaire à l'évolution du run-up (figure 3, panel d). Ces deux paramètres sont directement dépendants du cycle de marée, au cours duquel le niveau d'eau et la position de la zone de swash varient (figure 5).

Le paramètre d'Irribaren permet la prise en compte des conditions de vagues au large ainsi que de la pente de plage mais ne semble cependant pas proprement adapté à la caractérisation de la zone de swash. En effet, au cours du cycle de marée, la zone de surf est dissipative tandis que la zone de swash est considérée dissipative à marée basse $\left(\xi_{\sigma}<0.3\right)$ et intermédiaire à mi-marée et marée haute $\left(0.3<\xi_{\sigma}<1.5\right)$. Différentes définitions de la pente de plage ont été explorées, afin de caractériser au mieux la zone de swash, notamment en ce qui concerne la forme du profil de plage. A marée haute et basse, la plage du Truc Vert présente un profil de forme concave, contrairement à la mimarée ou celui-ci est convexe (figure 5). Ce changement de forme pourrait avoir un impact sur la dynamique du swash qu'il convient d'étudier plus en détail (figure 3, panel e). L'utilisation de la hauteur des vagues en zone de déferlement et non au large semblerait mieux adaptée à la paramétrisation du run-up (STOCKDON et al., 2006 ; GUEDES et al., 2011) mais ne suffit pas cependant à expliquer en totalité le comportement observé.

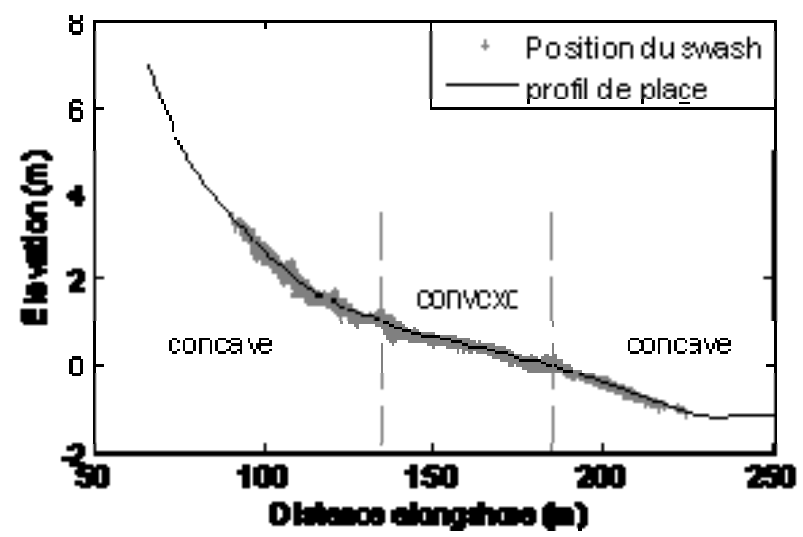

Figure 5. Profil de plage le long du transect étudié et position du swash le 27 mars 2008 au cours d'un cycle de marée. La barre externe est située plus au large 300 m.

\section{Conclusions}

Le run-up présente une modulation tidale importante qui ne semble pas être reliée aux conditions de vagues au large contrairement à ce qui est admis dans la littérature. Il 
semblerait que son comportement soit régi par différents facteurs hydrodynamiques et environnementaux tels la hauteur des vagues en zone de surf et la pente de plage, qui évoluent au cours du cycle de marée. L’influence des différents paramètres n'est cependant pas clairement établie, d'autant plus qu'ils évoluent au cours du cycle de marée et sont inter-corrélés. La définition de nouveaux paramètres prenant en compte la forme du profil de plage et la configuration de la zone de surf semble constituer une piste à explorer afin de caractériser au mieux le comportement de la zone de swash.

\section{Références bibliographiques}

BUTEL R., DUPUIS H., BONNETON P. (2002). Spatial variability of wave conditions on the french Atlantic coast using in-situ data. J. Coastal Res. SI 36, pp 96-108.

CASTELLE B., BONNETON P., DUPUIS H., SENECHAL N. (2007). Double bar beach dynamics on the high-energy meso-macrotidal French Aquitanian coast : a review. Marine Geology 245, pp 141-159. doi:10.1016/j.margeo.2007.06.001

GUEDES R.M.C., BRYAN K.R., COCO G., HOLMAN R. A. (2011). The effects of tides on swash statistics on an intermediate beach. J. of Geophys Res., 116, C04008, doi:10.1029/2010JC006660

GUZA R.T., THORNTON E.B. (1982). Swash oscillations on a natural beach. J. Geophys. Res., 87, pp 483-491. doi:10.1029/JC087iC01p00483

HOLMAN R.A., SALLENGER A.H. (1985). Setup and swash on a natural beach. J. Geophys. Res., 90, pp 945-953. doi:10.1029/JC090iC01p00945

RUESSINK B.G., KLEINHANS M.G., VAN DEN BEUKEL P.G.L. (1998). Observations of swash under highly dissipative conditions. J. Geophys. Res., 103, pp 3111-3118. doi:10.1029/97JC02791

RUGGIERO P., HOLMAN R.A., BEACH R.A. (2004). Wave run-up on a high-energy dissipative beach. J. Geophys. Res., 109, C0625. doi:10.1029/2003JC002160

SENECHAL N., ABADIE S., GALLAGHER E., MACMAHAN J.H.M., MASSELINK G., MICHALLET H., RENIERS J.H.M., RUESSINK B.G., RUSSELL P.E., SOUS D., TURNER I.L., ARDHUIN F., BONNETON P., BUJAN S., CAPO S., CERTAIN R., GARLAN T., PEDREROS R. (2011a). The ECORS- Truc Vert'08 nearshore field experiment: presentation of a three-dimensional morphologic system in a macro-tidal environment during consecutive extreme storm conditions. Ocean Dynamics. doi:10.1007/s10236-011-0472-x.

SENECHAL N., COCO G., BRYAN K.R., HOLMAN R.A. (2011b). Wave runup during extreme wave conditions. J. Geophys. Res. doi:10.1029/2010JC006814

STOCKDON H.F., HOLMAN R.A., HOWD P.A., SALLENGER JR. A.H. (2006). Empirical parameterizations of setup, swash and runup. Coast. Eng., 53, pp 573-588. doi:10.1016/j.coastaleng.2005.12.005 